\section{Wasserstoff löst keine Energieprobleme}

\author{
von Ulf Bossel, European Fuel Cell Forum
}

\begin{abstract}
Befürworter einer Wasserstoffwirtschaft sprechen von nachhaltiger Energie, die aus vielen Quellen abgeleitet werden kann. Diese Versprechungen sind kaum haltbar. Wasserstoff ist lediglich ein Energieträger, dessen Herstellung, Verteilung und Nutzung enorm viel Energie verschlingt. Selbst mit effizienten Brennstoffzellen kann man nur ein Viertel des ursprünglichen Energieinputs zurückgewinnen. Langfristig wird man Wasserstoff elektrolytisch mit Strom aus erneuerbaren Quellen erzeugen. Da sich Strom über Leitungen sehr effizient verteilen lässt, kann Wasserstoff den Wettstreit mit seiner Ursprungsenergie nie gewinnen. Aus physikalischen Gründen hat eine Wasserstoffwirtschaft keine Chance. Man sollte sich auf eine "Elektronenwirtschaft" einstellen.
\end{abstract}

\section{Energiezukunft}

Eigentlich ist die Energiezukunft deutlich erkennbar. Nach Versiegen der fossilen Vorräte muss die Menschheit ihren Energiebedarf aus erneuerbaren Quellen befriedigen. Statt chemischer Energie wird dann vorwiegend elektrische Energie von Wind-, Wasser- und Solarkraftwerken zur Verfügung stehen. Biomasse wird dieses Energieangebot ergänzen. Mit effizient genutztem Strom wird die Menschheit ihren Energiehunger ebenso gut decken können wie mit schlecht genutzten fossilen Brennstoffen oder künstlich erzeugtem Wasserstoff. Die Energiewirtschaft wird also auf den Kopf gestellt: Strom ist ausreichend verfügbar, aber chemische Träger werden zum kostbaren Luxus. Die Zukunft gehört der elektrischen Energie aus erneuerbaren Quellen.

Viele Befürworter einer nachhaltig geführten Energiezukunft möchten jedoch die chemische Energiewirtschaft beibehalten und fordern den zügigen Aufbau einer Wasserstoffwirtschaft. Die Umwandlung „grünen“ Stroms in Wasserstoff und dessen kommerzielle Nutzung sind jedoch mit hohen Verlusten verbunden. Wasserstoff und der daraus gewonnene Strom müssen deshalb immer wesentlich teurer sein als direkt verteilte Elektrizität. Wegen der hohen Verteilverluste erhöht sich auch die Zahl der Anlagen zur Gewinnung von Strom aus erneuerbaren Quellen. In einer nachhaltigen, auf Elektrizität aufbauenden Zukunft ist die Energieverteilung mittels Wasserstoff nur für wenige Sonderfälle zu rechtfertigen.

\section{Veränderungen im Energiebereich}

In Zukunft werden Solarstrahlung, Wind, Laufwasser, Wellen, Tidenhub, Geothermie und Biomasse geschätzte Energiequellen sein. Energie wird kostbar und deshalb mit höchster Effizienz verteilt und genutzt werden müssen. Eine nachhaltige Energiezukunft ruht deshalb auf zwei Pfeilern:

1. Energie aus erneuerbaren Quellen,

2. rationellste Energienutzung von der Quelle bis zur gewünschten Dienstleistung.

Mit Ausnahme von Biomasse und direkt genutzter Solarwärme wird Energie aus erneuerbaren Quellen jedoch als elektrischer Strom "geerntet". In einer nachhaltigen Energiezukunft werden fossile Energieträger durch Elektrizität verdrängt. Strom wird zur billigen Ausgangsenergie, während alle daraus künstlich erzeugten chemischen Energieträger - also auch Wasserstoff - teurer sein müssen. Strom wird so zur „Leitwährung“ des Energiesystems.

Die eiligst beschlossenen WasserstoffProgramme der Vereinigten Staaten und Europas verdeutlichen, dass die Dringlichkeit der Energieproblematik auch Politikern bewusst geworden ist. Der begleitende Paradigmenwechsel wird jedoch zu wenig beachtet. Während die heutige Energiewelt auf chemischen Energieträgern aufgebaut ist, wird eine zukünftige Energiewirtschaft vom Strom dominiert sein. Fossile Brennstoffe werden dann rar und teuer sein oder dürfen wegen der $\mathrm{CO}_{2}$-Emissionen nicht mehr eingesetzt werden. Aber Strom bleibt als "Quellenenergie" vorhanden. Damit werden thermische Kraftwerke oder Verbrennungsmotoren überflüssig, elektrische Wärmepumpen aber zu ,echten Energievermehrungsmaschinen“. Wasserstoff jedoch muss elektrolytisch durch Einsatz von Strom gewonnen werden. Dies macht ihn zur teuren „Luxusenergie“ für einige spezielle Anwen- 
dungen, nicht aber zum universellen Energieträger in einer allgemeinen Wasserstoffwirtschaft. Die Energiewelt muss sich auf Strom, nicht auf Wasserstoff vorbereiten. Die Wasserstoff-Programme könnten zu kostspieligen, zeitraubenden, aber erfolglosen Abenteuern werden.

\section{Zwischen heute und einer nachhaltigen Zukunft}

Trotz Globalisierung vollzieht sich der Übergang von einer fossilen zu einer nachhaltigen Energiewelt in allen Ländern und Regionen der Erde zeitlich versetzt und mit unterschiedlicher Geschwindigkeit. Die Ölförderländer werden als letzte erneuerbare Quellen erschließen, während sich Länder wie die Schweiz oder Dänemark schon deutlich in Richtung ,Naturenergie" bewegen, denn sie besitzen keine eigenen Quellen für Kohle, Öl oder Gas. Jede Region muss ihren eigenen Weg in die Energiezukunft finden. Der Umstieg kann nicht globalisiert werden. Europa wird Amerika in der Entwicklung vorauseilen.

Noch geht die Politik von der Dominanz der chemischen Energieträger aus und fordert den Ersatz von Kraftstoffen durch künstlich hergestellten Wasserstoff, also eine globale Wasserstoffwirtschaft. Diese Prämisse wird schon bald ihre Gültigkeit verlieren, denn aufgrund steigender Energiepreise bewegt sich der Energiebereich hin $\mathrm{zu}$ höherer Effizienz und damit auch hin zu elektrischen Systemen. Erst nach Abschluss dieser Phase wird die Zukunft planbar. Der übereilte Aufbau einer Wasserstoffwirtschaft ist mit hohen wirtschaftlichen Risiken verbunden und sollte deshalb gründlich bedacht werden.

Heute und in Zukunft wird sich die Energienutzung an das Energieangebot anpassen. Die Energiewirtschaft wird von mehreren Seiten bedrängt:

- Verbraucher reagieren auf steigende Energiepreise mit Gebäudesanierungen und sparsameren Fahrzeugen; der Anteil chemischer Energieträger am Gesamtverbrauch beginnt zu sinken.

- Durch Gebäudesanierungen wird der Heizwärmebedarf so stark reduziert, dass der
Einsatz von Elektroheizungen und Wärmepumpen vorteilhaft wird.

- Dreiliter-Autos, Kleinfahrzeuge, Dieselmotoren und Hybridfahrzeuge sind Vorboten einer neuen Mobilitätsstruktur; für die kurzen Fahrten zur Arbeit werden Kleinfahrzeuge mit physikalischen Energieträgern (Strom, Druckluft, Schwungrad) attraktiv.

- Unter guten meteorologischen Bedingungen liefern Sonne, Wind \& Co. bereits mehr Strom als den Betreibern thermischer Kraftwerke lieb ist; Strom verdrängt fossile Träger vom Energiemarkt.

Diese Trends führen zu einer graduellen Substitution chemischer Energieträger durch Elektrizität. Noch vor Einführung des Wasserstoffs werden wesentliche Teile des Energiesystems auf Strom umgestellt sein. Dann aber fehlt die Rechtfertigung für den Einsatz von Wasserstoff, denn mit wenigen Ausnahmen bietet die Originalenergie „Strom" im Vergleich zum Abkömmling „Wasserstoff" technische und wirtschaftliche Vorteile (siehe dazu insbes. Kap. 6).

Man muss deshalb genau überlegen, wie viel Wasserstoff in einer nachhaltig geführten Energiewirtschaft überhaupt benötigt wird und welche energetischen Prozesse nur mit Wasserstoff durchgeführt werden können. Zur Optimierung der energetischen Effizienz wird man möglichst viel Strom direkt und möglichst wenig für die Erzeugung von Wasserstoff einsetzen. Nur so kann der Energiebedarf aus erneuerbaren Quellen nachhaltig gedeckt werden.

\section{Energie für eine nachhaltige Energie- zukunft}

Erneuerbare Energie wird hauptsächlich als Elektrizität ,geerntet". Gleichstrom kommt von Solarzellen. Wind-, Wasser-, Wellen- und Gezeitenkraftwerke liefern Wechselstrom, ebenso auch solarthermische und geothermische Kraftwerke. Im nachfossilen Zeitalter werden lediglich Biomasse und organische Abfälle als chemische Primärenergie für die Herstellung flüssiger und gasförmiger Kohlenwasserstoffe zur Verfügung stehen. Der Fernverkehr - gleich ob zu Land, Wasser oder Luft - wird auch in $\mathrm{Zu}$ kunft nicht ohne flüssige, aus Biomasse hergestellte Kraftstoffe auskommen können. Wasser- 
stoff ist für diesen Einsatz ebenso ungeeignet wie elektrische Batterien.

Um diese erneuerbare Energie mit höchster Effizienz zu nutzen, müssen verlustreiche Wandlungsprozesse vermieden werden. Biomasse wird deshalb nicht in Kraftwerken ,verheizt", um Strom für die elektrolytische Wasserstoff-Erzeugung zu gewinnen. Man wird sie auch nicht zur Herstellung von Wasserstoff einsetzen, sondern in synthetische Kraftstoffe umwandeln, die wesentlich besser zu handhaben sind als das leichteste aller Gase. Aus energetischer Sicht ist die natürliche Bindung von Kohlenstoff an Wasserstoff eine wichtige Voraussetzung für die chemische Umwandlung von Biomasse in synthetische Kraftstoffe. Mit geringstem Energieaufwand vergären Bakterien nasse Biomasse $\mathrm{zu}$ gasförmigen oder flüssigen Energieträgern. Technische Prozesse, etwa das Fischer-Tropsch-Verfahren, stehen für die Umwandlung fester Stoffe zur Verfügung. Energetisch betrachtet ist die chemische Wandlung von Biomasse in Methan, Methanol, Ethanol oder Biodiesel der Wasserstoffgewinnung immer deutlich überlegen.

\section{Das Automobil als Trägheitsfaktor}

Nach Durchführung der vertretbaren Sparmaßnahmen könnte man den Energiebedarf aus erneuerbaren Quellen gut decken, wären da nicht die Bestrebungen rund ums Auto, an chemischen Energieträgern möglichst lange festzuhalten. Automobil- und Ölgesellschaften haben ein verständliches Interesse an chemischen Energieträgern für die eingeführte Antriebstechnik. Der Einsatz von Wasserstoff und Brennstoffzelle ist im Grunde eine technische Variante von Benzin und Ottomotor, nur mit einem anderen chemischen Energieträger.

Leider folgt die Politik den Argumenten der etablierten Lobby. Die „Hydrogen Initiative“ und das „FreedomCar"-Programm von Präsident Bush finden Nachahmer in aller Welt. Die Wasserstoff-Diskussion lenkt jedoch ab von dringend benötigten Maßnahmen im Energiebereich. Energieprobleme können nur durch Erschließung erneuerbarer Quellen und rationelle Energienutzung gelöst werden, nicht aber durch Wechsel des chemischen Energieträgers.
In der Tat, einige Aspekte der Wasserstoffwirtschaft sind bestechend. So bezaubert die Idee, dass aus Wasserstoff und Luft ohne Ausstoß von Treibhausgasen oder Ozonkillern nur sauberer Wasserdampf entsteht. Auch die Elektrolyse frappiert. Man nehme Strom und Wasser und erhält Wasserstoff. Besondere Begeisterung findet die 1838 vom Basler Chemiker Christian Friedrich Schönbein entdeckte und vom Engländer William Robert Grove 1842 verwirklichte Brennstoffzelle. Die verbreitete Faszination von Wasserstoff und Brennstoffzelle sind verständlich. Veränderungen im Energiebereich müssen jedoch auf physikalischen Fakten und technischen Erkenntnissen basieren und nicht auf faszinierenden Beobachtungen und naivem Wunschdenken.

\section{Wasserstoffwirtschaft ist Energie- verschwendung}

Die relativ günstige Gewinnung von Wasserstoff aus Erdgas ist keine gute Eintrittskarte zur Wasserstoffwirtschaft, denn nach Versiegen der fossilen Quellen wird man Wasserstoff nur noch durch die elektrolytische Spaltung von Wasser erzeugen können. Dafür wird Gleichstrom benötigt und zwar viel mehr, als selbst mit bester Technik jemals aus dem erzeugten Brenngas zurück gewonnen werden kann. Bei der Elektrolyse wird elektrische Energie in chemische Energie umgewandelt. Wasserstoff ist also keine Energiequelle, sondern lediglich ein Sekundärenergieträger, vergleichbar mit dem Wasser in einer Zentralheizung.

Von der Quelle bis zur Nutzung gleicht der Weg des Wasserstoffs einem steilen Abstieg ins Tal. Jede Stufe der Energiekette ist mit Energieverlusten bzw. -aufwand verbunden (vgl. Abb. 1 nächste Seite). Bei Wasserstoff sind die Energieverluste jedoch so groß, dass dem Wasserstoffverbraucher hinter einer effizienten Brennstoffzelle nur noch ein Viertel der elektrischen Primärenergie zur Verfügung steht. Nur ein Viertel des erneuerbaren Stroms wird genutzt, während drei Viertel ungenutzt verloren gehen. Diese Verluste sind physikalisch bedingt und können auch durch zusätzliche Forschungen nicht wesentlich verringert werden. 
Abb. 1: Die Energievernichtungskaskade der Wasserstoffwirtschaft

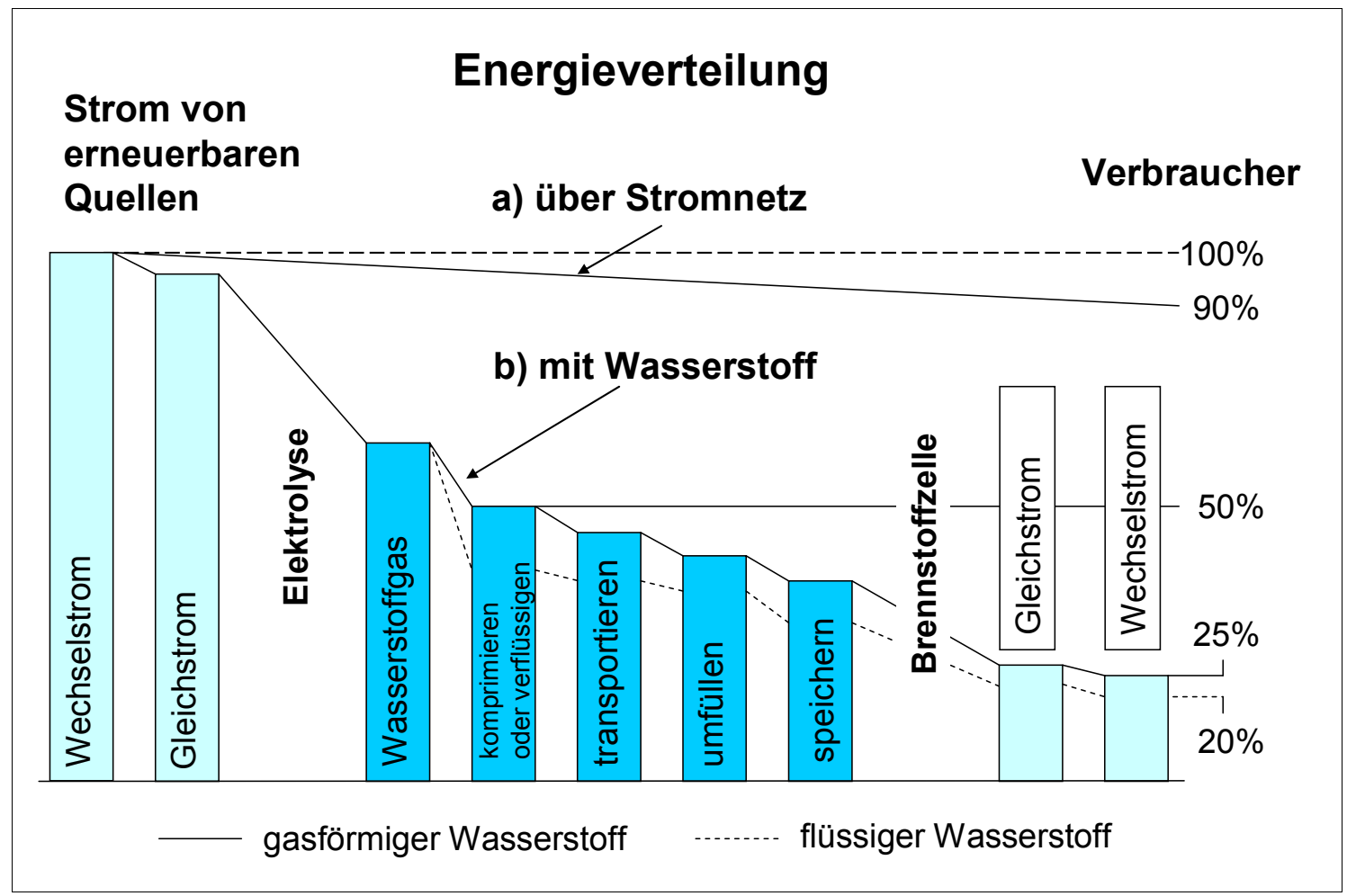

Tab. 1: Typischer Energiebedarf für Erzeugung, Kompression bzw. Verflüssigung, Transport und Nutzung von Wasserstoff

\begin{tabular}{|l|l|c|c|}
\hline Verfahrensschritte & \multicolumn{1}{|c|}{ Technische Angaben } & $\begin{array}{c}\text { Energieverluste } \\
M J / \mathrm{kg} \mathrm{H} H_{2}\end{array}$ & $\begin{array}{c}\text { Verluste } \\
\text { (in \% des } \mathrm{H}_{o} \text { Wasserstoff) }\end{array}$ \\
\hline Elektrolyse & $1,76 \mathrm{Volt}, 1 \mathrm{~atm}$ & 61 & $43 \%$ \\
\hline Kompression & $1 \mathrm{bar}-200 \mathrm{bar}$ & 10 & $7 \%$ \\
\hline & $1 \mathrm{bar}-400 \mathrm{bar}$ & 17 & $9 \%$ \\
\hline & $1 \mathrm{bar}-800 \mathrm{bar}$ & 65 & $12 \%$ \\
\hline Verflüssigung & $100 \mathrm{~kg} / \mathrm{h}$ & 45 & $32 \%$ \\
\hline & $1.000 \mathrm{~kg} / \mathrm{h}$ & 35 & $28 \%$ \\
\hline Straßentransport & $10.000 \mathrm{~kg} / \mathrm{h}$ & 18 & $13 \%$ \\
\hline & $200 \mathrm{bar}, 200 \mathrm{~km}$ & 36 & $25 \%$ \\
\hline & $200 \mathrm{bar}, 400 \mathrm{~km}$ & 3 & $2 \%$ \\
\hline Pipeline & flüssig, $200 \mathrm{~km}$ & 12 & $8 \%$ \\
\hline $\begin{array}{l}\mathrm{H}_{2} \text {-Elektrolyse an Tank- } \\
\text { stelle }\end{array}$ & 10 bar, $1.000 \mathrm{~km}$ & $80 *$ & $56 \%$ \\
\hline Betanken & entspricht 60.000 Liter Benzin & 5 & $4 \%$ \\
\hline Brennstoffzelle & 100 bar auf 400 bar & 142 & $50 \%$ \\
\hline
\end{tabular}

einschl. Wasseraufbereitung, Kompression, Gebäudeenergiebedarf usw.; der Brennwert $\left(\mathrm{H}_{\mathrm{o}}\right)$ von Wasserstoff beträgt $142 \mathrm{MJ} / \mathrm{kg}$

Quelle: Bossel et al. 2003 
Der interne Energiebedarf der Wasserstoffwirtschaft ist eingehend analysiert worden (Bossel et al 2003). In Tabelle 1 (s. vorhergehende Seite) sind der für die wichtigsten Wandlungsschritte benötigte Energiebedarf in absoluten Energieeinheiten MJ (Megajoule) pro kg Wasserstoff oder in Prozent der im Wasserstoff enthaltenen Energiemenge (Brennwert oder oberer Heizwert $\mathrm{H}_{\mathrm{o}}=142 \mathrm{MJ} / \mathrm{kg}$ ) angegeben.

Zugegeben, auch die heutige Energieversorgung ist nicht frei von Verlusten. Zwischen Ölquelle und Tankstelle werden 8 bis $12 \%$ der sprudelnden Energie für Förderung, Raffinierung und Transport benötigt. Bei Wasserstoff gehen jedoch von der Elektrolyse bis zur Wasserstofftankstelle mindestens $50 \%$ verloren oder müssen für Kompression, Verflüssigung, Transport, Umfüllen usw. aufgewendet werden. Noch schlimmer wird es bei einer Rückverstromung des Wasserstoffs. Selbst mit effizienten Brennstoffzellen stehen dem Verbraucher nur etwa $25 \%$ des ursprünglich eingesetzten Stroms zur Nutzung zur Verfügung. Bei direkter Verteilung könnte der Verbraucher jedoch etwa $90 \%$ des Stroms nutzen. Die in Abb. 1 gezeigte Verlustkaskade ist für die Wasserstoffwirtschaft repräsentativ.

\section{Die Besonderheiten des Wasserstoffs}

Wasserstoff ist kein Gas wie jedes andere. Aufgrund seiner physikalischen Merkmale unterscheidet er sich deutlich von Erdgas (Methan) oder Luft. Wasserstoff ist 8-mal leichter als Methan (Erdgas) und 15-mal leichter als Luft. Um diese Gase auf 200 bar zu komprimieren benötigt man etwa 13,8 MJ / kg für Wasserstoff, $1,4 \mathrm{MJ} / \mathrm{kg}$ für Methan, aber nur $0,9 \mathrm{MJ} / \mathrm{kg}$ für Luft. Bei Wasserstoff verschlingt die Kompression etwa $9 \%$, bei Methan nur 2,5\% des Energieinhalts des jeweiligen Gases. Bei 200 bar sind in einem Liter Wasserstoff 2,6 MJ, in Erdgas jedoch 8,0 MJ oder über dreimal mehr Energie enthalten. Benzin enthält mit 33,8 MJ / Liter sogar 13-mal mehr Energie als der auf 200 bar komprimierte Wasserstoff. Brennstoffzellenautos fahren mit Wasserstoff (Tankdruck 350 bar) gerade einmal $250 \mathrm{~km}$ weit. Bei gleichem Druck, aber mit Erdgas befüllt, würde die getankte Energie im Erdgasauto Fahrten bis 500 $\mathrm{km}$ erlauben. Wasserstoff lässt sich am besten als Kohlenwasserstoff speichern oder transpor- tieren. Ein Kubikmeter flüssiger Wasserstoff wiegt nur $70 \mathrm{~kg}$ und ist damit etwas schwerer als Styropor. Aber ein Kubikmeter Benzin wiegt $700 \mathrm{~kg}$ (und enthält $128 \mathrm{~kg}$ Wasserstoff). Entsprechend unterschiedlich ist der obere Heizwert $\left(\mathrm{H}_{\mathrm{o}}\right)$ : 33,8 MJ für den Liter Benzin, aber nur 9,9 MJ für den Liter Flüssigwasserstoff.

Diese und andere physikalischen Merkmale machen reinen Wasserstoff nur bedingt geeignet für den täglichen Einsatz als Energieträger. Ein Tanklastwagen kann $26 \mathrm{t}$ Benzin transportieren. Für dieselbe Energiemenge an Wasserstoff benötigt man etwa 22 mit Wasserstoffdruckflaschen (300 bar) beladene Wasserstofftransporter. Wegen des geringen Energieinhalts des Wasserstoffs werden pro $100 \mathrm{~km}$ Lieferdistanz über $6 \%$ der gelieferten Energiemenge konsumiert. Bei Benzin sind es nur gerade einmal $0,2 \%$. Für die Verteilung von flüssigem Wasserstoff ist der spezifische Energiebedarf für den Transport niedriger. Dafür werden jedoch mindestens $30 \%$ des Energieinhalts für die Verflüssigung des Wasserstoffs benötigt. Eine sinnvolle Lösung für den Straßentransport von Wasserstoff ist daher nicht in Sicht.

Im Gegensatz dazu kann man regenerativ gewonnenen Strom über Leitungen verteilen, denn es gibt keinen besseren Weg als den direkten Energietransport mit Elektronen. In modernen Netzen wird elektrische Energie mit einem Wirkungsgrad von über $90 \%$ umweltfreundlich vom Kraftwerk zu Verbrauchern transportiert.

Die Befürworter einer Wasserstoffwirtschaft schlagen deshalb vor, Wasserstoff an Tankstellen vor Ort elektrolytisch zu erzeugen. Eine Autobahntankstelle, die heute täglich etwa 60.000 Liter Benzin oder Diesel verkauft, hätte einen elektrischen Leistungsbedarf von mindestens 26 Megawatt und würde 107 Kubikmeter Wasser täglich benötigen. Die zuverlässige Erfüllung dieser Kriterien rund um die Uhr und zu allen Jahreszeiten ist für viele Standorte auf der Welt kein einfaches Problem. Auch der Energietransport per Pipeline erfordert für Wasserstoff etwa viermal mehr Energie als für Erdgas. Aus verschiedenen Gründen sind sowohl die bestehenden Erdgasleitungen als auch die Erdgasinfrastruktur nicht für den Transport von Wasserstoff geeignet. Diese Fakten zeigen, wie sehr sich Wasserstoff von Erdgas unterscheidet und wie neuartig die Probleme einer Wasserstoffwirtschaft sind. 


\section{Energiekosten in einer Wasserstoff- wirtschaft}

Auch in Zukunft werden Verluste mit den Energiepreisen verrechnet. Da lediglich $50 \%$ des für die Elektrolyse benötigten Stroms beim Verbraucher als nutzbarer Wasserstoff angeliefert würden, dürfte die im Wasserstoffgas enthaltene Energie mindestens doppelt so teuer sein wie elektrische Energie aus der Steckdose. Heizen mit Strom wäre billiger als Heizen mit Wasserstoff. Erdgas wird deshalb kaum durch Wasserstoff ersetzt werden. Heizöl und Erdgas würden durch Wärmedämmung und elektrische Heizgeräte ersetzt, nicht aber durch Wasserstoff.

Die Verteuerung gilt auch für die Stromerzeugung, denn selbst mit effizienten Brennstoffzellen kann man lediglich $50 \%$ der im Wasserstoff enthaltenen Energie in elektrische Energie umwandeln. Aus Wasserstoff erzeugter Strom muss deshalb mindestens viermal teurer sein als Netzstrom. Angesichts der deutlich günstigeren direkten elektrischen Lösung kann die Stromerzeugung mit Wasserstoff und Brennstoffzellen nie attraktiv werden. Jeder Versuch, Strom in Form von Wasserstoff zu vermarkten, ist aus ökonomischen Gründen zum Scheitern verurteilt. Es gibt kein Nebeneinander von Wasserstoff und Strom, denn Wasserstoff an sich erfüllt noch keine Kundenwünsche. Der Verbraucher benötigt Strom aus der Brennstoffzelle.

Diese Argumente gelten auch für mobile Anwendungen. In einer von Elektrizität dominierten nachhaltig geführten Energiewirtschaft werden Fahrzeuge mit elektrischem Antrieb attraktiv, denn pro Energieeinheit kostet Strom aus der eigenen Steckdose wesentlich weniger als Wasserstoff an der Tankstelle. Auch sind Elektromobile mit Rückgewinnung der Bremsenergie äußerst effizient. Bis zu $80 \%$ der ,getankten" elektrischen Energie werden den Rädern zugeführt. Im Brennstoffzellen-Fahrzeug wird der doppelt so teure Wasserstoff jedoch nur mit einem Tank-Rad-Wirkungsgrad von etwa $40 \%$ genutzt. Ein mit Wasserstoff-Brennstoffzelle ausgestattetes Fahrzeug benötigt etwa viermal mehr Strom als ein Elektrofahrzeug, bzw. verursacht viermal höhere Kraftstoffkosten. Für Fahrten zur Arbeit wird man vermutlich Elektrofahrzeuge mit Hybridantrieb verwenden. Mit Lithium-Ionen-Batterien ausgestattete Elektrofahrzeuge fahren bereits bei ca. 1,2 1/ $100 \mathrm{~km}$
Benzinäquivalent mit einer Batterieladung $250 \mathrm{~km}$ weit und sind bei einem leistungsstarken Anschluss in wenigen Minuten aufgeladen. Als Lebensdauer dieser Batterien werden zehn Jahre genannt. Brennstoffzellenautos können diese Werte heute auch schon erreichen, wenn auch bei wesentlich höheren Betriebskosten.

Für größere Distanzen, für den Last-, Luftund Seetransport wird man dagegen synthetische, aus Biomasse hergestellte Kraftstoffe einsetzen. Bei vergleichbar niedriger Umweltbelastung übertreffen diese Energieträger den Wasserstoff bezüglich Handhabbarkeit, Energiedichte, Sicherheit und Kosten. Eine übereilte Einführung des Wasserstoffs im Verkehrsbereich ist angesichts dieser sich abzeichnenden Entwicklungen mit hohen wirtschaftlichen Risiken behaftet.

\section{Woher Wasser und Energie für Wasserstoff?}

Die Konsequenzen einer Wasserstoffwirtschaft werden mit folgendem Beispiel verdeutlicht. Zur Herstellung von einem Kilogramm Wasserstoff (Energieinhalt von 3,5 Litern Benzin) werden $9 \mathrm{~kg}$ Wasser sowie (einschließlich Elektrolyse, Verflüssigung, Transport, Lagerung und Verteilung) etwa $100 \mathrm{kWh}$ Strom benötigt. Wie viel Wasser und Strom werden benötigt, um den Flugverkehr in Frankfurt/Main auf Wasserstoff umzustellen?

Am Frankfurter Flughafen werden täglich 50 Jumbo-Jets mit je 130 Tonnen $\left(160 \mathrm{~m}^{3}\right)$ Flugbenzin befüllt. Die gleiche Energiemenge steckt in 50 Tonnen $\left(715 \mathrm{~m}^{3}\right)$ flüssigem Wasserstoff. Zur Betankung aller Jumbos mit Wasserstoff müssten täglich $2.500 \mathrm{~m}^{3}$ Flüssigwasserstoff bereitgestellt werden, für dessen Herstellung man $22.500 \mathrm{~m}^{3}$ sauberes Wasser und die elektrische Leistung von acht Kraftwerken von je $1 \mathrm{GW}$ benötigt (zum Vergleich: Das AKW Biblis hat eine Leistung von 1,3 GW). Für die Versorgung aller Flugzeuge des Flughafens mit Wasserstoff müsste man den Wasserverbrauch der Stadt Frankfurt und die Energie von mindestens 25 Großkraftwerken einsetzen.

Die Energie für den Wasserstoff stammt eben nicht einfach nur aus ,erneuerbaren Quellen", wie das die Befürworter einer Wasserstoffwirtschaft gerne darstellen. Die Fragen „Woher das Wasser?“ und „Woher der Strom?“ 
müssen vor dem Aufbau neuer Infrastrukturen zuerst einmal beantwortet werden. Es gibt viele Gegenden in der Welt, in denen das spärlich fließende Wasser zur Erhaltung menschlichen Lebens verwendet werden sollte.

\section{Wohin führt der Weg?}

Der Übergang von der heutigen, vom Erdöl dominierten Energiewirtschaft zu einer nachhaltigen, von regenerativ erzeugtem Strom geprägten basiert also nicht auf einer einfachen Substitution fossiler Energieträger durch synthetischen Wasserstoff. Komplexe Veränderungen müssen in allen Bereichen der Energietechnik bedacht werden: Erzeugung, Verteilung, Speicherung und Nutzung sind in jedem Fall zu berücksichtigen. Die Energiewirtschaft wird auf den Kopf gestellt. Während chemische Energieträger heute die Ausgangsbasis bilden, wird es in Zukunft Strom aus erneuerbaren Quellen sein. Heute ist Elektrizität die sekundäre Energieform, morgen ist es der künstliche erzeugte Energieträger Wasserstoff. Während heute Erdgas und Erdöl preisbestimmend sind, wird es in Zukunft Strom aus regenerativen Quellen sein. Strom wird zur „Leitwährung“ im Energiemarkt. Der aus Strom gewonnene Wasserstoff wird deshalb immer teurer sein als die regenerativ erzeugte Elektrizität. Daran lässt sich nicht rütteln, weder mit politischen Entscheidungen noch mit aufwändigen Entwicklungsprogrammen.

In einer nachhaltig geführten Energiewirtschaft wird synthetischer Wasserstoff deshalb keine wichtige Rolle spielen, denn ,Strom direkt" liefert fast immer bessere Lösungen. Der Sekundärenergieträger Wasserstoff kann sich nicht gegen den Energieträger Strom durchsetzen, aus dem er künstlich erzeugt wurde. In der nachhaltigen Welt verlieren Umweltargumente ebenfalls ihre Gültigkeit, denn elektrischer Strom ist ebenso sauber wie der daraus gewonnene Wasserstoff. Im Gegenteil: Wegen der Ineffizienz der Wasserstoffkette müsste man zur Bereitstellung der benötigten Endenergie wesentlich mehr regenerative Kraftwerke errichten als bei einer direkten Stromverteilung und Nutzung. Umweltschützer beklagen sich heute schon über die Verschandelung des Landschaftsbildes durch Windkraftanlagen. Bei einer Energieverteilung mittels Wasserstoff würde sich die Zahl der Windräder vervierfa- chen. Wer stellt dafür die Landschaft zur Verfügung? Woher sollen die Energie für die Erzeugung des Wasserstoffs und das benötigte Wasser kommen? Eine quantifizierte Antwort auf diese Frage müssen die Befürworter der Wasserstoffwirtschaft erst noch liefern.

Ein übereilter Einstieg in eine Wasserstoffwirtschaft wird den Übergang zur Nachhaltigkeit nicht nur stark behindern, sondern vielleicht sogar unmöglich machen. Für alle Befürworter einer nachhaltigen Zukunft hat die Erschließung neuer Energiequellen eindeutig Vorrang vor der Einführung eines neuen Energieträgers. Dazu gehört auch die Beschäftigung mit der Frage, wie viele Kraftwerke (gleich ob Wind oder Atom) die Menschheit akzeptieren kann. Die Zukunft verlangt markante Veränderungen im Energiebereich. Man sollte mit Mut und Zielstrebigkeit eine nachhaltige Energiewelt direkt ansteuern, statt zuerst einmal mit einer Wasserstoffwirtschaft $\mathrm{zu}$ experimentieren. Der Weg führt zur effizienten Nutzung von Elektrizität aus erneuerbaren Quellen. Eine Wasserstoffwirtschaft hat keine Zukunft.

\section{Literatur}

Bossel, U.; Eliasson, B.; Taylor, G., 2003: The Future of the Hydrogen Economy: Bright or Bleak? Final Report, April 15 ${ }^{\text {th }}, 2003$

\section{Kontakt}

Ulf Bossel

European Fuel Cell Forum

Morgenacherstraße $2 \mathrm{~F}$

5452 Oberrohrdorf, Schweiz

Tel.: +41 - 56 - $496-7292$

Fax: +41 - 56 - 496 - 4412

E-Mail: forum@efcf.com

Internet: http://www.efcf.com 